\title{
Inscribing a regular $\boldsymbol{m}$-gon in a regular $\boldsymbol{n}$-gon
}

\author{
S. J. Dilworth and S. R. Mane
}

\begin{abstract}
We find necessary and sufficient conditions on $m$ and $n$ for inscribing a regular $m$-gon in a regular $n$-gon.

Mathematics Subject Classification (2010). Primary 51M20; Secondary $52 \mathrm{C} 15$.
\end{abstract}

Keywords. Inscribed polygons, polygon containment, axes of symmetry.

\section{Introduction}

Suppose that $P$ and $Q$ are closed regular polygons. We say that $P$ is inscribed in $Q$ if every vertex of $P$ lies on the perimeter of $Q$. Note that $P$ and $Q$ are not assumed to be concentric or to have a common axis of symmetry. We present the necessary and sufficient conditions on $m$ and $n$ for inscribing a regular $m$-gon in a regular $n$-gon. Naïvely we expected this problem to be solved in the time of Euclid, but it seems to be not completely solved. The solutions for inscribing triangles and squares in polygons are well-known, (see, e.g., puzzles by Martin Gardner in Scientific American [3], specifically for the case of a square or rectangle in a triangle) and also the case where $m$ divides $n$ is obvious. However these are not the only possible cases. We present a statement of the complete solution to the problem:

Theorem. Suppose that $m, n \geq 3$. A regular $m$-gon can be inscribed in a regular $n$-gon if and only if one of the following mutually exclusive conditions is satisfied:

(a) $m=3$;

(b) $m=4$;

(c) $m \geq 5$ and $m$ divides $n$;

(d) $m \geq 6$ is even and $n$ is an odd multiple of $m / 2$. (Note that this includes the case $n=m / 2$.)

In cases (c) and (d) the polygons are necessarily concentric and in case (d) they share a common axis of symmetry. In case (d) we insist that $n$ be an odd 
multiple of $m / 2$ because if $n$ is an even multiple of $m / 2$, then $n$ is a multiple of $m$, which is already covered in case (c).

Our proof of the result is based on a simple application of the Pigeon-hole Principle.

\section{Solution of problem}

Let $Q$ be a regular $n$-gon $(n \geq 5)$ in the complex plane with edges of unit length. We assume that $Q$ is positioned in the upper half-plane so that $z=0$ is a vertex and the $y$-axis is the axis of symmetry of $Q$ through that vertex. We use arc length $s$ measured counterclockwise from $z=0$ to identify the perimeter of $Q$ with the real interval $[0, n)$. Under this identification $0,1, \ldots, n-1$ are the vertices of $Q$ and $[r, r+1](r=0, \ldots, n-1)$ are the closed edges of $Q$. We say that a point $s$ on the perimeter of $Q$ is of type $a(0 \leq a<1)$ if $s-a \in \mathbb{N}$, i.e., if $s$ and $a$ are in the same relative positions on their respective edges.

The proof of the theorem requires two elementary lemmas. The first is intuitively obvious, but for the sake of completeness we include a proof in the next section. (Here $\phi_{n}:=(n-2) \pi / n$ is the internal angle of a regular $n$-gon.)

Lemma 2.1. Suppose that $n \geq 5$ and $3 \pi / 5 \leq \phi<\pi$. For each point $A$ on the perimeter of $Q$ (unless $A$ is a vertex of $Q$ and $\phi \geq \phi_{n}$ ) there exist unique points $B$ and $C$ on the perimeter of $Q$ such that $|A B|=|A C| \neq 0$ and $\widehat{C A B}=\phi$. (If $A$ is a vertex of $Q$ and $\phi=\phi_{n}$ then $B$ and $C$ are not unique, while if $A$ is a vertex of $Q$ and $\phi>\phi_{n}$ then such points $B$ and $C$ do not exist.)

Suppose that $\min \{m, n\} \geq 5$. For $n>m$, by Lemma 2.1 for each $s \in[0, n)$ on the perimeter of $Q$ there exist unique points $p(s)$ and $q(s)$ on the perimeter of $Q$ such that the chords joining $s$ to $p(s)$ and $s$ to $q(s)$ have equal length and subtend an angle $\phi_{m}$. We label $p(s)$ and $q(s)$ so that the triple $[s, p(s), q(s)]$ is positively oriented. Let $f(s)$ denote the length of the chord joining $s$ to $p(s)$. Clearly, $f(s)$ is a continuous function of $s \in[0, n)$ and has period one. Note that by symmetry $f(s)=f(1-s)$ for $0 \leq s \leq 1$.

For $m>n$, using Lemma 2.1 again, we can define $f(s)$ as above provided $s$ is not a vertex. Note that if $s$ is a vertex then $f(s)$ is undefined since $Q$ clearly does not admit chords of equal length subtending an angle $\phi_{m}$ at a vertex (as $\left.\phi_{m}>\phi_{n}\right)$. However, setting $f(s):=0$ when $s$ is a vertex again defines a continuous periodic function on [0,n). Finally, if $A$ is a vertex of $Q$ and $\phi_{m}=\phi_{n}$ then $m=n$. We exclude this trivial case below.

The theorem follows easily from the next lemma and the Pigeon-hole Principle. We postpone the slightly technical proof of the lemma until the next section.

Lemma 2.2. For $\min \{m, n\} \geq 5$ and $m \neq n$ the level sets $L(y):=\{s \in$ $[0,1): f(s)=y\}(y \geq 0)$ have cardinality at most four. 


\section{Proof of the theorem.}

Case 1: $m=3$ or $m=4$. The case $m=3$ is obvious. Indeed, for every point $s$ on the perimeter of $Q$ there is a unique equilateral triangle inscribed in $Q$ which has a vertex at $s$. Figure 1 a $(m, n)=(3,7)$ exhibits equilateral triangles, with and without a common axis of symmetry, inscribed in a heptagon.

For $m=4$ there is (by compactness) a largest square $P$ contained in $Q$ which shares the $y$-axis with $Q$ as an axis of symmetry. By symmetry, $P$ must have

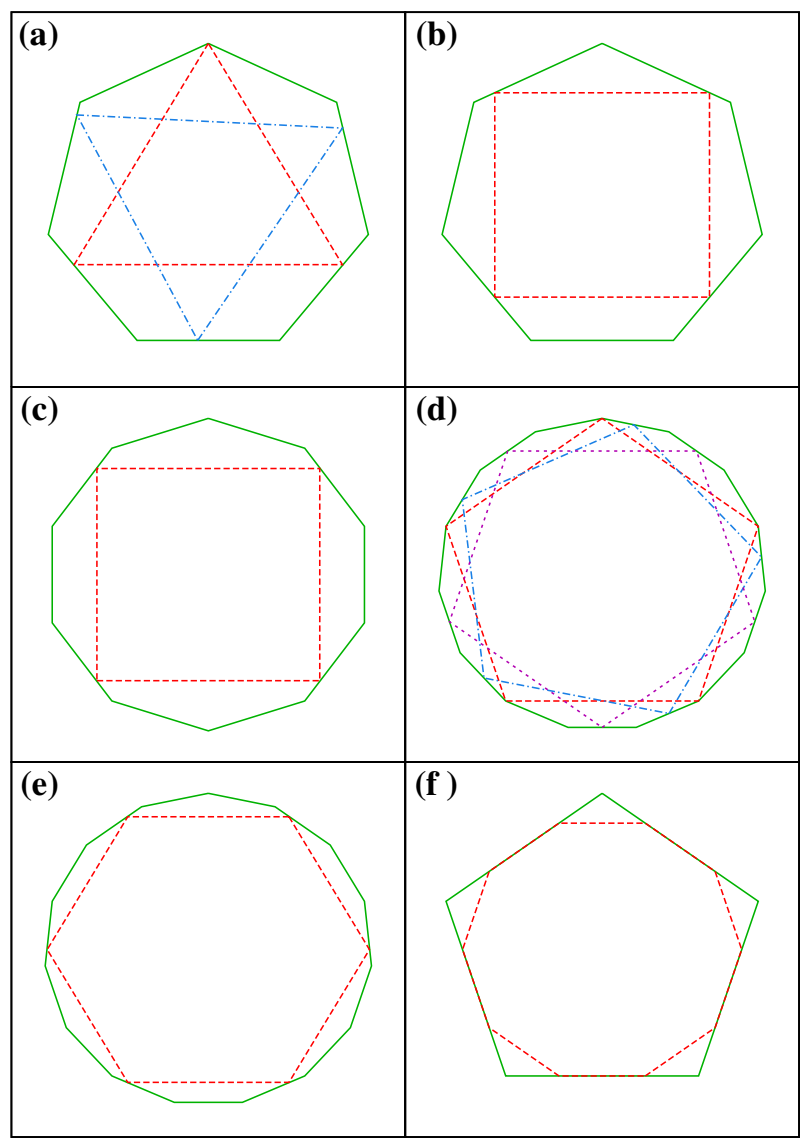

Figure 1 Plots of illustrative examples: a $(m, n)=(3,7)$ : inscribed triangles with (dash) and without (dotdash) shared axis of symmetry; b $(4,7)$ : inscribed square, shared symmetry axis but not concentric; c $(4,10)$ : inscribed square, shared pair of symmetry axes and concentric; $\mathbf{d}(5,15)$ : inscribed pentagons maximal (dash), minimal (dotted) and generic (dotdash); e $(6,15)$ : inscribed hexagon, three shared symmetry axes and concentric; f $(10,5)$ : inscribed decagon, $n=m / 2$ ('snipped off vertices') 
an even number points of contact with the perimeter of $Q$ that are symmetric about the $y$-axis. If there were only two points of contact then $P$ could be translated parallel to the $y$-axis so that there were no points of contact, but this would contradict the maximality of $P$. So $P$ is inscribed in $Q$. Note that in general $P$ and $Q$ are not concentric. Figure $1 \mathrm{~b}(m, n)=(4,7)$ illustrates the non-concentric case and Fig. $1 c(m, n)=(4,10)$ the concentric case, where $P$ and $Q$ actually share two symmetry axes.

Note: After submitting this paper, J. Cantarella from the University of Georgia kindly brought to our attention additional results for equilateral triangles and squares inscribed in planar closed curves. See in particular the papers by Emch [1] and [2], where in the latter paper Emch proves that any closed convex rectilinear polygon admits at least one inscribed square.

Case 2: $5 \leq m<n$. We continue to use the notation introduced above. Suppose that $P$ is a regular $m$-gon inscribed in $Q$. Note that if $s$ is a vertex of $P$ then its adjacent vertices in $P$ are $p(s)$ and $q(s)$, so $f(s)$ is constant on the vertices of $P$. Since $m \geq 5$ and by Lemma 2.2 the level sets of $\left.f(s)\right|_{[0,1]}$ have cardinality at most four, it follows from the Pigeon-hole Principle that two vertices of $P$ have the same type.

Note that the type of $s$ determines the types of $p(s)$ and $q(s)$. Thus, if two adjacent vertices of $P$ have the same type, then all the vertices of $P$ have the same type (and hence $P$ and $Q$ are concentric). But this implies that the number of vertices of $Q$ cut off by any edge of $P$ is constant, and hence $m$ divides $n$. This yields condition (c). It is obvious that (c) is also a sufficient condition. Figure $1 \mathrm{~d}(m, n)=(5,15)$ illustrates the smallest, the largest, and an intermediate pentagon inscribed in a 15 -gon.

Suppose that there are two vertices $r$ and $s$ of $P$ that have type $a$, say, and that no two adjacent vertices of $P$ are of the same type. Then $p(r)$ and $p(s)$ both have the same type $b$, say, where $a \neq b$. Since $r$ and $s$ have the same type, the centre of $Q$ lies on the perpendicular bisector of the chord joining $r$ to $s$. Similarly, the centre of $Q$ lies on the perpendicular bisector of the chord joining $p(r)$ to $p(s)$. Hence $P$ and $Q$ are concentric with common centre located at the point of intersection of these perpendicular bisectors. This implies that $b=1-a$ (since only points of type $1-a$ are at the same distance as points of type $a$ from the centre of $Q$ ) and that the types of the vertices of $P$ alternate between type $a$ and type $1-a$. But this implies that $m$ is even, $m=2 k$, say. Since the number of vertices of $Q$ cut off by a chord joining any vertex of $P$ of type $a$ to the next vertex of $P$ of type $a$ (these two vertices have a common neighbouring vertex of type $1-a$ ) is constant, it follows that $k$ divides $n$. Hence the number of vertices of $Q$ cut off by edges of $\mathrm{P}$ alternates between $t$ and $t+r$, where $t \geq 0$ and $r \geq 0$ are fixed, so $n=(2 t+r) k$. Only the case $r=1$ (i.e. $n$ is an odd multiple of $k$ ) would give chords of $Q$ of equal length (which is a necessary and sufficient condition for $P$ to be regular). To see that $r=1$ gives case $(\mathrm{d})$, suppose that $n=(2 t+1) k$. Then the Intermediate Value Theorem yields a choice of $0<a<1$ corresponding to chords of $Q$ of equal length such 
that the number of vertices of $Q$ cut off by adjacent chords alternates between $t$ and $t+1$ (note that the case $t=0$ corresponds to the case $m=2 n$ discussed below). Figure 1e illustrates the case $(m, n)=(6,15)$.

Case 3: $5 \leq n<m$. The argument is very similar to the case $m<n$, so we can be brief. For $m \geq 6$ Lemma 2.2 and the Pigeon-hole Principle again imply that two vertices of $P$ are of the same type. Since $m>n$ it is clear that every edge of $Q$ contains at least one vertex of $P$ and that some edge of $Q$ contains two vertices of $P$, so $n+1 \leq m \leq 2 n$. In particular, it is not possible for all the vertices of $P$ to be of the same type. Arguing as above, we must have $m=2 n$ with the types of the vertices of $P$ alternating as $a$ and $1-a$ for some $0<a<1 / 2$. To see that $m=2 n$ is also a sufficient condition, note that $P$ can be obtained from $Q$ by 'snipping off' the vertices of $Q$. Figure 1f shows a decagon inscribed in a pentagon.

Remark 2.3. The proof of the theorem actually determines the configuration of $P$ and $Q$ in cases (c) and (d). In case (c) the vertices of $P$ are of the same type (Fig. 1d). In case (d) the types of the vertices alternate, which implies that the lines joining midpoints of pairs of opposite edges of $P$ are common axes of symmetry of $P$ and $Q$ (Fig. 1e, f).

\section{Proofs of the lemmas}

Proof of Lemma 2.1. We may assume that $A$ lies on the edge $[n-1,0)$. First we examine the case $\phi<\phi_{n}$. For $\theta \geq-\pi / n$ consider a chord which subtends an angle $\theta$ with the positive $x$-axis and joins $A$ to a point $B_{\theta}$ on the edge $[r, r+1)$. It is easily verified that $(r-1) \pi / n \leq \theta<(r+1) \pi / n$ and that the directed edge $[r, r+1]$ subtends an angle $(2 r+1) \pi / n$ with the positive $x$-axis. Hence the chord length $\left|A B_{\theta}\right|$ is a strictly increasing function of $\theta$ for $-\pi / n \leq \theta \leq(r+1) \pi / n$ provided $(2 r+1) \pi / n \leq(r-1) \pi / n+\pi / 2$, i.e., $r+1 \leq[n / 2]-1$.

Now suppose that $A B_{\theta}$ is one of two chords which subtend an angle $\phi$ at $A$. Then $\theta \leq(n-1) \pi / n-\phi \leq 2 \pi / 5-\pi / n$ (since $\phi \geq 3 \pi / 5)$. To ensure that $\left|A B_{\theta}\right|$ is increasing in the range $-\pi / n \leq \theta \leq 2 \pi / 5-\pi / n$ we require $2 \pi / 5-\pi / n \leq([n / 2]-1) \pi / n$, which is valid for $n \geq 5$. Let $C_{\theta}$ be the point on the perimeter of $Q$ such that $C_{\theta} \hat{A} B_{\theta}=\phi$. We showed above that $\left|A B_{\theta}\right|$ increases (and consequently $\left|A C_{\theta}\right|$ decreases) as $\theta$ increases. Hence by the Intermediate Value Theorem there is a unique value $\theta_{0} \in[-\pi / n, 2 \pi / 5-\pi / n]$ such that $\left|A B_{\theta_{0}}\right|=\left|A C_{\theta_{0}}\right|$. Set $B:=B_{\theta_{0}}$ and $C:=C_{\theta_{0}}$.

Some small modifications to the proof are required in the case $\phi \geq \phi_{n}$. For the existence of $B$ and $C$ (or uniqueness in the case $\phi=\phi_{n}$ ) we require $A$ not to be a vertex. Moreover, for $\phi>\phi_{n}$, when $A$ is sufficiently close to the vertex $n-1$ then $A$ and $B$ both lie on the edge $[n-1,0]$, i.e. $\theta_{0}=-\pi / n$. Similarly, when $A$ is sufficiently close to the vertex 0 then $A$ and $C$ both lie on the edge $[n-1,0]$. 
Finally, we prove Lemma 2.2. Two further lemmas are required, of which the first is of independent interest.

Lemma 3.1. Let $L_{1}:=\left\{t \exp i \psi_{1}: t \in \mathbb{R}\right\}$ and $L_{2}:=\left\{t \exp i \psi_{2}: t \in \mathbb{R}\right\}$ be two lines passing through the origin and let $0<\phi<\pi$.

(a) Suppose that $\sin \left(\phi+\psi_{1}-\psi_{2}\right) \neq 0$. Then for each $z \in \mathbb{C}$ there exist unique points $u(z) \in L_{1}$ and $v(z) \in L_{2}$ such that the directed line segments $[z, u(z)]$ and $[z, v(z)]$ have equal length and subtend an angle $\phi$, i.e.

$$
v(z)-z=\exp (i \phi)(u(z)-z) .
$$

Moreover, setting $F_{\phi}(z):=|z-u(z)|$, then, for all $z_{0}, w_{0} \in \mathbb{C}, F_{\phi}^{2}\left(t z_{0}+\right.$ $\left.w_{0}\right)$ is a non-negative quadratic function of $t \in \mathbb{R}$.

(b) Suppose $\sin \left(\phi+\psi_{1}-\psi_{2}\right)=0$. Then, for each $z \in \mathbb{C}$, either $u(z)$ and $v(z)$ satisfying (3.1) do not exist or for every $u(z) \in L_{1}$ there exists $v(z) \in L_{2}$ satisfying (3.1).

Proof. Let $u(z)=u \exp i \psi_{1}$ and $v(z)=v \exp i \psi_{2}$. Then, setting $z=x+i y$ and taking real and imaginary parts, we obtain the following pair of linear equations for $u$ and $v$ :

$$
\begin{aligned}
& -u \cos \left(\phi+\psi_{1}\right)+v \cos \psi_{2}=x(1-\cos \phi)+y \sin \phi, \\
& -u \sin \left(\phi+\psi_{1}\right)+v \sin \psi_{2}=-x \sin \phi+y(1-\cos \phi) .
\end{aligned}
$$

These equations have a unique solution for every choice of $z$ if and only if $\sin \left(\phi+\psi_{1}-\psi_{2}\right) \neq 0$, and in that case there exist $b, c \in \mathbb{C}$, depending only on $\phi, \psi_{1}$, and $\psi_{2}$, such that $u(z)=b z+c \bar{z}$. Hence

$$
F_{\phi}^{2}\left(t z_{0}+w_{0}\right)=\left|t\left(z_{0}-b z_{0}-c \overline{z_{0}}\right)+\left(w_{0}-b w_{0}-c \overline{w_{0}}\right)\right|^{2},
$$

which is a non-negative quadratic function of $t$. This proves (a). On the other hand, if $\sin \left(\phi+\psi_{1}-\psi_{2}\right)=0$, then the linear equations are degenerate, and (b) follows easily.

Henceforth we shall refer to the function $F_{\phi}(z)$ as the $\phi$-distance determined by $L_{1}$ and $L_{2}$.

Lemma 3.2. Suppose that $n>m \geq 5$ and that $p(0)$ is not a vertex of $Q$. Then $f_{+}^{\prime}(0) \geq 0$, where $f_{+}^{\prime}(0):=\lim _{s \downarrow 0}(f(s)-f(0)) / s$ is the right-hand derivative.

Proof. By symmetry neither $q(0)$ nor $p(0)$ is a vertex of $Q$. Let $p(0)$ lie on the open edge $(r, r+1)$. Then $r \pi / n<\pi / m<(r+1) \pi / n$ and the directed edge $[r, r+1]$ subtends an angle $(2 r+1) \pi / n$ with the positive $x$-axis. Let us show that this angle is not obtuse. If $n \leq 10$ then it is easily checked that $(2 r+1) \pi / n \leq \pi / 2$, with equality only if $m=5$ and either $n=6$ or $n=10$. If $n \geq 11$, then

$$
(2 r+1) \pi / n<2 \pi / m+\pi / n \leq 2 \pi / 5+\pi / 11<\pi / 2 .
$$


By symmetry $q(s)$ lies on the edge $(n-r-1, n-r)$. Let $L_{1}$ and $L_{2}$ be the extensions of the edges $(r, r+1)$ and $(n-r-1, n-r)$, let $F_{\phi_{m}}(z)$ be the $\phi_{m^{-}}$ distance determined by $L_{1}$ and $L_{2}$, and let $u(z)$ be defined as in Lemma 3.1. (Since $p(0)$ and $q(0)$ are uniquely defined, it follows from Lemma 3.1 that $F_{\phi_{m}}(z)$ is well-defined.) By symmetry, $\frac{\partial F_{\phi_{m}}}{\partial x}(0)=0$. Since the directed edges $[r, r+1]$ and $[n-r-1, n-r]$ subtend a non-obtuse angle with the positive $x$-axis, it follows that $|u(i y)-i y|$ is an increasing function of $y$, and hence $\frac{\partial F_{\phi_{m}}}{\partial y}(0) \geq 0$. Since $p(0)$ and $q(0)$ are interior points of their respective edges, it follows that $f(s)=F_{\phi_{m}}(s \cos \pi / n, s \sin \pi / n)$ for all sufficiently small $s>0$. Hence $f_{+}^{\prime}(0)=\sin (\pi / n) \frac{\partial F_{\phi_{m}}}{\partial y}(0) \geq 0$.

Proof of Lemma 2.2. First we examine the case $n>m \geq 5$. If $p(0)$ is not a vertex of $Q$, then as $s$ increases from 0 to $1, p(s)$ (resp., $q(s)$ ) moves counterclockwise from $p(0)$ (resp., $q(0)$ ) to the point $p(1)$ (resp., $q(1)$ ) of the same type on an adjacent edge, passing through a vertex of $Q$ in transit. Hence there exist $0<s_{0} \leq s_{1}<1$, where $s_{1}=1-s_{0}$, such that the restrictions

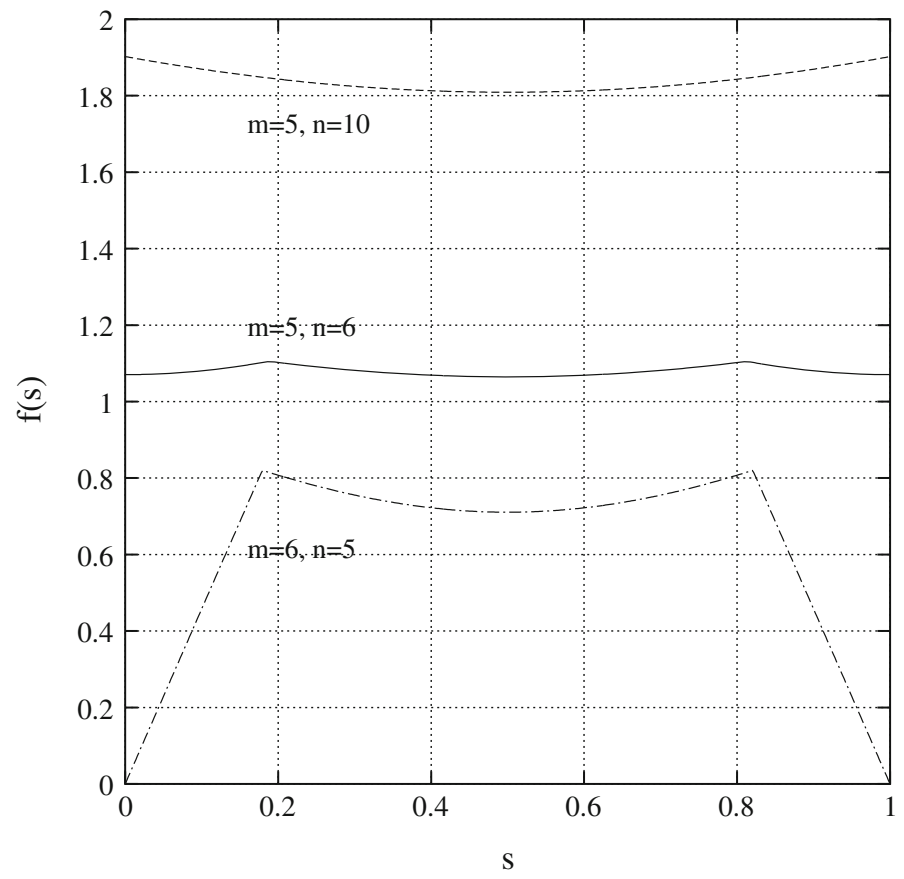

Figure 2 Graphs of $f(s)$ for $0 \leq s \leq 1$ for three representative cases: (a) $(m, n)=(5,6)$, i.e. $n>m \geq 5$ and $p(0)$ not a vertex of $Q$, (b) $(m, n)=(5,10)$, i.e. $n>m \geq 5$ and $p(0)$ a vertex of $Q$, and (c) $(m, n)=(6,5)$, i.e. $m>n \geq 5$. The definitions of $f(s)$ and $p(0)$, etc., are given in the text 
of $f(s)$ to each of the intervals $\left[0, s_{0}\right],\left[s_{0}, s_{1}\right]$, and $\left[s_{1}, 1\right]$ are the $\phi_{m}$-distances determined by corresponding pairs of edges (extended to be pairs of lines); e.g., $\left.f\right|_{\left[s_{0}, s_{1}\right]}$ is the $\phi_{m}$-distance determined by the edges containing $p(1 / 2)$ and $q(1 / 2)$. By Lemma 3.1 the restriction of $f^{2}(s)$ to each of these subintervals is a non-negative quadratic function of $s$. By Lemma 3.2, $f_{+}^{\prime}(0) \geq 0$, and hence the graph of $\left.f^{2}(s)\right|_{\left[0, s_{0}\right]}$ is a strictly increasing segment of a parabola. By symmetry, $\left.f^{2}(s)\right|_{\left[s_{1}, 1\right]}$ is strictly decreasing. Lastly, the graph of $\left.f^{2}(s)\right|_{\left[s_{0}, s_{1}\right]}$ is a segment of a parabola with a local minimum at $s=1 / 2$. It follows that each level set $L(y)$ has cardinality at most four. An example of a graph of $f(s)$ for $0 \leq s \leq 1$ is shown as the solid curve in Fig. 2, for $m=5$ and $n=6$. On the other hand, if $p(0)$ is a vertex of $Q$, then $\left.f^{2}(s)\right|_{[0,1]}$ is a non-negative quadratic function, and hence the level sets have cardinality at most two. Note that in this case $m$ must divide $n$. An example of a graph of $f(s)$, where $p(0)$ is a vertex of $Q$, is shown as the dashed curve in Fig. 2, for $m=5$ and $n=10$.

Finally, we consider the case $m>n \geq 5$. Recall that in this case $f(0)$ is undefined since $Q$ does not admit chords of equal length subtending an angle $\phi_{m}$ at a vertex (as $\phi_{m}>\phi_{n}$ ). However, setting $f(0):=0$, then arguing as above we see that the restrictions of $f(s)$ to intervals $\left[0, s_{0}\right],\left[s_{0}, s_{1}\right]$, and $\left[s_{1}, 1\right]$ (where $s_{0}$ and $s_{1}$ are as defined above) are linear, hyperbola (square root of a quadratic), and linear, respectively. Hence the level sets $L(y)$ again have cardinality at most four. An example of a graph of $f(s)$ is shown as the dotdash curve in Fig. 2, for $m=6$ and $n=5$.

\title{
Acknowledgements
}

S. J. Dilworth was supported by NSF Grant DMS 0701552. We thank M. Ghomi and S. Tabachnikov for their helpful comments and suggestions. We also thank J. Cantarella for informing us of the work of Emch.

\section{References}

[1] Emch, A.: Some properties of closed convex curves in a plane. Am. J. Math. 35, 407-412 (1913)

[2] Emch, A.: On the medians of a closed convex polygon. Am. J. Math. 37, 19-28 (1915)

[3] Gardner, M.: see for example a list of mathematical puzzles at http://www. ansible.co.uk/misc/mgardner.html

\author{
S. J. Dilworth \\ Department of Mathematics \\ University of South Carolina \\ Columbia, SC 29208, USA \\ e-mail: dilworth@math.sc.edu
}


S. R. Mane

Convergent Computing Inc.

P.O. Box 561

Shoreham, NY 11786, USA

e-mail: srmane001@gmail.com

Received: December 7, 2009. 